\title{
Riemann Zeta Invariance Under Composed Integral Transform
}

Benedict Irwin ${ }^{1}$

${ }^{1}$ University of Cambridge

January 7, 2021

From a question I asked online [1], I had deduced that the Laplace transform could be absorbed into the inverse Mellin transform as

$$
\mathcal{L} \mathcal{M}^{-1}[\phi]=-\mathcal{M}^{-1}\left[\phi^{*}\right]
$$

and the Mellin transform could be absorbed into the inverse Laplace transform as

$$
\mathcal{M L}^{-1}[\psi]=\Gamma(q) \mathcal{L}^{-1}\left[\psi^{*}\right]
$$

where

$$
\phi^{*}=\Gamma(t) \phi(1-t)
$$

and

$$
\psi^{*}=\psi\left(-e^{-s}\right) e^{-s}
$$

the term of $\phi(1-t)$ reminded me of the Riemann function equation for $\zeta(s)$ which is

$$
\zeta(s)=2^{s} \pi^{s-1} \sin \left(\frac{\pi s}{2}\right) \Gamma(1-s) \zeta(1-s)
$$

the question I was then interested in was what other transform when applied to the inverse Mellin transform of a function, would result in this functional equation, or what is the transform such that $\zeta(s)$ is invariant to?

The more fundamental quantity in terms of Mellin transforms is $\Gamma(s) \zeta(s)$ which has the integral (Mellin transform) representation:

$$
\Gamma(s) \zeta(s)=\int_{0}^{\infty} \frac{x^{s-1}}{e^{x}-1} d x=\mathcal{M}\left[\frac{1}{e^{x}-1}\right]
$$

we would like to find an integral transform of a function $f \mathcal{Q}[f]$ such that

$$
\mathcal{Q}\left[\mathcal{M}^{-1}[\phi(s)]\right]=\mathcal{M}^{-1}\left[2^{s} \pi^{s-1} \sin \left(\frac{\pi s}{2}\right) \Gamma(s) \phi(1-s)\right]
$$

such that

$$
\mathcal{Q}\left[\mathcal{M}^{-1}[\Gamma(s) \zeta(s)]\right]=\mathcal{M}^{-1}[\Gamma(s) \zeta(s)]
$$

by virtue of the integral equation 6 we should have something like

$$
\mathcal{Q}\left[\frac{1}{e^{x}-1}\right](s)=\frac{1}{e^{s}-1}
$$

we expect $\mathcal{Q}$ to somewhat resemble a Laplace transform because of the equation

$$
\mathcal{L M}^{-1}[\phi(s)]=\mathcal{M}^{-1}[\Gamma(s) \phi(1-s)]
$$


Seems that we want something such that

$$
\mathcal{Q}\left[x^{-s}\right]=\left(\frac{q}{2 \pi}\right)^{s-1} \frac{1}{\pi} \sin \left(\frac{\pi}{2}(1-s)\right) \Gamma(1-s)
$$

the trick seems to be using an inverse Mellin transform on the above to get the relationship

$$
Q\left[x^{-s}\right]=\int_{0}^{\infty} x^{-s} \frac{\sin \left(\frac{q}{2 \pi x}\right)}{\pi x} d x=q^{-s} 2^{s} \pi^{s-1} \Gamma(s) \sin \left(\frac{\pi s}{2}\right)
$$

this is still not quite right as we want to invert the $s \rightarrow 1-s$. It does seem (numerically) for a small region of $s$ values (between 0 and 1?) that

$$
\mathcal{Q}\left[x^{-s}\right]=\int_{0}^{\infty} x^{-s} \frac{\sin \left(\frac{q x}{2 \pi}\right)}{\pi} d x=\left(\frac{q}{2 \pi}\right)^{s-1} \frac{1}{\pi} \sin \left(\frac{\pi}{2}(1-s)\right) \Gamma(1-s)
$$

as required. Hence our transform becomes (note the minus sign)

$$
\mathcal{Q}[f]=-\int_{0}^{\infty} f(x) \frac{\sin \left(\frac{q x}{2 \pi}\right)}{\pi} d x
$$

which should (formally) satisfy

$$
\mathcal{Q}\left[\mathcal{M}^{-1}[\Gamma(s) \zeta(s)]=\mathcal{M}^{-1}[\Gamma(s) \zeta(s)]\right.
$$

or then 'fixing' the inverse Mellin transform as given, $\Gamma(s) \zeta(s)$ is some kind of eigen-function of the transform $\mathcal{Q} \ldots$

\section{Checking This Follows Through}

Thus

$$
\begin{gathered}
\mathcal{Q}\left[\mathcal{M}^{-1}[\phi]\right]=\mathcal{Q}\left[\frac{1}{2 \pi i} \int_{c-i \infty}^{c+i \infty} x^{-s} \phi(s) d s\right] \\
\mathcal{Q}\left[\mathcal{M}^{-1}[\phi]\right]=\frac{1}{2 \pi i} \int_{c-i \infty}^{c+i \infty} \mathcal{Q}\left[x^{-s}\right] \phi(s) d s \\
\mathcal{Q}\left[\mathcal{M}^{-1}[\phi]\right]=\frac{-1}{2 \pi i} \int_{c-i \infty}^{c+i \infty}\left(\frac{q}{2 \pi}\right)^{s-1} \frac{1}{\pi} \sin \left(\frac{\pi}{2}(1-s)\right) \Gamma(1-s) \phi(s) d s
\end{gathered}
$$

by letting $s-1 \rightarrow-t$ we get

$$
\mathcal{Q}\left[\mathcal{M}^{-1}[\phi]\right]=\frac{1}{2 \pi i} \int_{c^{\prime}-i \infty}^{c^{\prime}+i \infty} q^{-t} 2^{t} \pi^{t-1} \sin \left(\frac{\pi t}{2}\right) \Gamma(t) \phi(1-t) d t=\frac{1}{2 \pi i} \int_{c^{\prime}-i \infty}^{c^{\prime}+i \infty} q^{-t} \phi^{*}(t) d t=\mathcal{M}^{-1}\left[\phi^{*}\right]
$$

where $\phi^{*}(t)=2^{t} \pi^{t-1} \sin \left(\frac{\pi t}{2}\right) \Gamma(t) \phi(1-t)$. If we set $\phi(t)=\Gamma(t) \zeta(t)$ according to the Riemann functional equation we have

$$
\zeta(s)=2^{s} \pi^{s-1} \sin \left(\frac{\pi t}{2}\right) \Gamma(1-s) \zeta(1-s)
$$

thus $\phi^{*}(t)=\phi(t)=\Gamma(t) \zeta(t)$.

\section{Conclusion}

It is formally possible to define such an integral transform. This may be possible and have better convergence for other functional relationships. 


\section{References}

[1] - https://math.stackexchange.com/questions/2501698/a-pair-of-composed-integral-transforms-frommellin-and-laplace-transforms

\section{Appendix}

If we define the forward transform as

$$
\mathcal{Q}_{1}[f(x)](k)=\int_{0}^{\infty} \frac{f(x)}{e^{k x}-1} d x
$$

we find that

$$
\mathcal{Q}_{1}\left[x^{s-1}\right](k)=k^{-s} \Gamma(s) \zeta(s), s>1, t>0
$$

or equivalently

$$
\mathcal{Q}_{1}\left[x^{s-1}\right](k)=k^{-s} 2^{s} \pi^{s-1} \sin \left(\frac{\pi s}{2}\right) \Gamma(s) \Gamma(1-s) \zeta(1-s), s>1, t>0
$$

Thus

$$
\begin{gathered}
\mathcal{Q}_{1}\left[\mathcal{M}^{-1}[\phi]\right]=\mathcal{Q}_{1}\left[\frac{1}{2 \pi i} \int_{c-i \infty}^{c+i \infty} x^{-s} \phi(s) d s\right] \\
\mathcal{Q}_{1}\left[\mathcal{M}^{-1}[\phi]\right]=\frac{1}{2 \pi i} \int_{c-i \infty}^{c+i \infty} \mathcal{Q}_{1}\left[x^{-s}\right] \phi(s) d s \\
\mathcal{Q}_{1}\left[\mathcal{M}^{-1}[\phi]\right]=\frac{1}{2 \pi i} \int_{c-i \infty}^{c+i \infty} q^{s-1} \Gamma(1-s) \zeta(1-s) \phi(s) d s
\end{gathered}
$$

by letting $s-1 \rightarrow-t$ we get

$$
\mathcal{Q}_{1}\left[\mathcal{M}^{-1}[\phi]\right]=\frac{-1}{2 \pi i} \int_{c^{\prime}-i \infty}^{c^{\prime}+i \infty} q^{-t} \Gamma(t) \zeta(t) \phi(1-t) d t=\frac{-1}{2 \pi i} \int_{c^{\prime}-i \infty}^{c^{\prime}+i \infty} q^{-t} \phi^{*}(t) d t=-\mathcal{M}^{-1}\left[\phi^{*}\right]
$$

where $\phi^{*}(t)=\Gamma(t) \zeta(t) \phi(1-t)$. Although this is cool, it's not quite what we want. 